\title{
Symposium
}

\section{Surgical management of carcinoma of gall bladder}

\author{
Col A.K. Tyagi \\ Senior Advisor (Surgery \& Surgical Oncology), Base Hospital, Delhi Cantt-10, India
}

\section{Introduction}

Gall bladder (GB) cancer is an aggressive tumor causing early spread and death. It spreads early by lymphatic metastasis, hematogenous metastasis, and direct infiltration into liver and also has a high propensity to seed the peritoneal surface after tumor spillage and seeding of biopsy tract and laparoscopic ports. Classically 5 year survival in most cases is less than $5 \%$ and median survival is less than 6 months. ${ }^{1}$ GB cancer has highest incidence in Indians, Pakistanis, Chileans, Bolivians, Central Europeans, Israelis, American Indians. ${ }^{2}$

\section{Etiology}

(a) Gall stones - 75-98\% of all patients with gall bladder cancer have cholelithiasis. Although cholelithiasis and chronic inflammation of the GB are involved in the pathogenesis of this tumor, the incidence of GB cancer in a population of patients with gall stones is only $0.3-3 \%$. Chronic exposure seems to act as a promoter for some other carcinogenic exposure.

(b) Porcelain gall bladder (calcification of GB) - is associated with cancer in $10-25 \%$ of cases.

(c) APBDJ - predisposes to GB malignancy.

(d) Helicobacter bilis and pylori infection - of GI increases biliary tract carcinoma by six fold. ${ }^{3}$

\section{Mode of spread}

Gall bladder has a thin wall, a narrow lamina propria and only a single muscle layer. Once it penetrates the thin muscle layer, it has access to major lymphatics and vascular channels. GB cancer thus tends to have early lymphatic and hematogenous spread. At autopsy, GB cancer patient have 91-94\% incidence of lymphatic metastasis, $65-82 \%$ of hematogenous spread and $60 \%$ of peritoneal spread. ${ }^{4}$ The lymphatic drainage descends down around the bile duct involving cystic and pericholedochal nodes first. From there connections are made to nodes posterior to pancreas, portal vein and common hepatic artery. Finally, the flow reaches the outer aortocaval, celiac and superior mesenteric nodes. Lymph never ascends towards hepatic hilum. Some connections are made directly from the pericholedochal nodes to interaortocaval nodes which make it difficult to control this disease with a regional lymph node dissection. ${ }^{5}$ Hematogenous metastasis tends to be from invasion of small veins extending directly from the GB onto the portal venous system, leading to hepatic metastasis in segment IV and V of the liver.

\section{Clinical presentation}

The clinical presentation of gall bladder cancer is akin to biliary colic or chronic cholecystitis. Persistent right upper quadrant pain associated with recent weight loss in elderly patients should be suspected to having a GB cancer. Lump in right upper quadrant is a frequent clinical presentation. Jaundice and anorexia are signs of advanced disease and so is ascites. Some GB cancers are discovered intra-operatively in patients undergoing cholecystectomy for cholelithiasis, or on HPE following cholecystectomy.

\section{Investigations}

(a) Laboratory investigations - liver function tests, including assessment of hepatic reserve.

(b) Tumor markers - Ca19-9 and CEA can be considered although these markers are not specific for GB cancer. ${ }^{6}$ CA19-9 may aid in the diagnosis in suspicious clinical situations, but is not cost effective for general screening of patients undergoing cholecystectomy.

(c) Imaging.

E-mail address: oncodocsurgeon@hotmail.com. 0377-1237/\$ - see front matter ( 2012 , Armed Forces Medical Services (AFMS). All rights reserved. doi:10.1016/j.mjafi.2012.04.007 
USG - is the most common initial radiologic investigation for right upper quadrant pain. Discontinuous GB mucosa, echogenic mucosa, submucosal echolucency favors malignancy. A polypoid mass was present in $27 \%$ and a GB replacing invasive mass was present in $50 \%$ of cases of GB cancer examined. $^{7}$

CT scan - reveals a mass obliterating the GB lumen in $42 \%$ of cases, a polypoidal mass in $26 \%$ and a diffuse wall thickening in $6 \%$ of cases. However, only one-third of pathologically positive nodes are identified preoperatively by CT-scan.

Endoscopic ultrasound and guided biopsy - may be a helpful adjunct to other imaging modalities for evaluation of periportal and peripancreatic adenopathy.

MRCP (magnetic resonance cholangiopancreatography) is required in patients presenting with jaundice to evaluate biliary and hepatic invasion by tumor. It is preferred over ERCP (endoscopic retrograde cholangiopancreatography) or PTC (percutaneous transhepatic cholangiography) being noninvasive unless stenting is planned.

PET scanning - has not been established in the evaluation of patients with GB cancer though there is emerging evidence that it picks up distant metastatic disease in patients with otherwise potentially resectable disease.

Chest imaging - to rule out pulmonary metastasis.

(d) Tissue diagnosis (biopsy and cytology).

Cholecystectomy prior to definitive resection, as a diagnostic biopsy is unacceptable. One should be prepared for definitive resection at the time of initial exploration.

(a) Bile cytology - diagnosis by examination of bile cytology for malignant cells avoids isolating the tumor and seeding of malignant cells in peritoneal cavity or abdominal wound. Any patient suspected of having GB cancer undergoing ERCP or PTC should have bile cytology for malignant cells. The sensitivity of bile cytology alone for diagnosis of GB cancer is between 50 and $73 \%{ }^{8}$

(b) Core needle biopsy/percutaneous FNAC - should be performed for masses that are not considered for surgical resection. Core needle biopsy has a higher chance of resulting in needle tract seeding than FNAC and should be kept in reserve for cases in which FNAC is inconclusive.

(c) Endoscopic ultrasound directed FNAC - for gall bladder lesion has $80 \%$ sensitivity and $100 \%$ specificity and is probably the best option for obtaining tissue diagnosis. ${ }^{9}$

\section{Staging (TNM)}

Primary tumor

$\mathrm{T} 1-\mathrm{T} 1 \mathrm{a}-$ invasion of lamina propria

$\mathrm{T} 1 \mathrm{~b}$ - invasion of muscularis propria

T2 - invasion of perimuscular connective tissue; no extension beyond serosa or into liver

T3 - perforation of serosa/or direct invasion of liver and/or one other adjacent organ or structure, e.g. stomach, duodenum, colon, pancreas, omentum, and extrahepatic bile ducts.
T4 - invasion of main portal vein or hepatic artery, or invasion of two or more extrahepatic organs or structures.

\section{Regional lymph nodes}

NO - no lymph node involvement.

$\mathrm{N} 1$ - involvement of regional lymph nodes.

The regional lymph nodes are the cystic duct node and the pericholedochal, hilar, peripancreatic (head only), periduodenal, periportal, celiac and superior mesenteric nodes.

\section{Metastases}

M0 - no distant metastases.

M1 - distant metastases present.

\section{Stage grouping}

Stage I A - T1, N0, M0.

I B - T2, N0, M0.

Stage II A - T3, N0, M0.

IIB - T1-3, N1, M0.

Stage III - T4, N0-1, M0.

Stage IV - any T, any N, M1.

\section{Surgery for gall bladder cancer}

Prophylactic cholecystectomy - for asymptomatic cholelithiasis to prevent development of carcinoma is not indicated since the incidence of GB cancer is low compared with the incidence of the cholelithiasis in the population.

High risk situations may benefit from prophylactic cholecystectomy, e g - patients with APBDJ and normal size bile duct, North Indian women with asymptomatic cholelithiasis, ${ }^{10}$ porcelain GB.

Whether the prophylactic surgery should be laparoscopic or open? In high risk situations serum CA 19.9 evaluation and bile cytology will be helpful in making a preoperative diagnosis of cancer. Laparoscopic surgery could be reserved for those with normal markers and negative cytology. For those highly suspicious of cancer, open approach is preferred for fear of inadvertent seeding of the peritoneal cavity.

Simple cholecystectomy - Stage 1a tumor can be treated with simple cholecystectomy. Any suspicious node should be sent for frozen section examination.

Extended (radical) cholecystectomy - Stage 1b, II and selected stage III (T4, N0) tumor should be treated with en bloc resection of GB, segments $\mathrm{IVb}$ and $\mathrm{V}$ of liver and regional lymph node dissection with or without bile duct excision.

Amount of liver resection has ranged from a limited wedge excision of $2 \mathrm{~cm}$ of liver around the gall bladder bed to routine extended right hepatic lobectomy. The goal is to achieve a negative margin on the tumors, encompassing cells that have directly infiltrated the liver. 
Lymphadenectomy should include the lymph nodes at the porta hepatis, gastro-hepatic ligament and retro duodenal regions. Nodal disease outside of this area (mostly celiac, retropancreatic or in the inter aorto-caval region) should be considered unresectable.

If the tumor is in the GB neck and there is suspicion of mucosal spread into the common bile duct or inflammation and scarring compromises adequate skeletonization of the porta hepatis, then resection of the common bile duct with Roux-en-Y hepaticojejunostomy should be done.

\section{Conflicts of interest}

None identified.

\section{R E F E R E N C E S}

1. Perpetuo MD, Valdivieso M, Heibrun LK, et al. Natural history of gallbladder cancer: a review of 36 years experience at M.D. Anderson hospital and Tumor Institute. Cancer. 1978;42:330.

2. Kiran RP, Polaka N, Dudrick SJ. Incidence pattern and survival for gall bladder cancer over three decades; an analysis of 10301 patients. Ann Surg Oncol. 2007;14:827.
3. Matsukura N, Yokomuro S, Yamada S, et al. Association between Helicobacter bilis in bile and biliary tract malignancies: $\mathrm{H}$. bilis in bile from Japanese and Thai patients with benign and malignant diseases in the biliary tract. Jpn J Cancer Res. 2002;93:842.

4. Kimura W, Nagai H, Kuroda A, et al. Clinicopathologic study of asymptomatic gall bladder carcinoma found at autopsy. Cancer. 1989;64:98.

5. Shirai Y, Yoshida K, Tsukada K, et al. Identification of the regional lymphatic system of the gall bladder by vital staining. Br J Surg. 1992;79:659.

6. Koda M, Yashima K, Kawaguchi K, et al. Expression of Fhit, Mlh1, and p53 protein in human gall bladder carcinoma. Cancer Lett. 2003;199:131.

7. Wibbenmeyer LA, Sharafuddin MJ, Wolverson MK, et al. Sonographic diagnosis of unsuspected gall bladder cancer: imaging findings in comparison with benign gall bladder conditions. AJR Am J Roentgenol. 1995;165:1169.

8. Akosa AB, Barker F, Desa L, et al. Cytologic diagnosis in the management of gall bladder carcinoma. Acta Cytol. 1995;39:494.

9. Meara RS, Jhala D, Eloubeidi MA, et al. Endoscopic ultrasound guided FNA biopsy of bile duct and gall bladder; analysis of 53 cases. Cytopathology. 2006;17:42.

10. Mohandas KM, Patil PS. Cholecystectomy for asymptomatic gallstones can reduce gall bladder cancer mortality in northern Indian women. Indian J Gastroenterol. 2006;25:816 